\title{
Viral communities associated with porcine diarrhoeal disease and genetic characterization of a bufavirus in Tibetan pigs in China
}

\author{
Long Zhou ${ }^{1}$ (D) Chengzhe Hu ${ }^{1} \cdot$ Qun Zhou ${ }^{1} \cdot$ Danjiao Yang ${ }^{2} \cdot$ Lixuan Wang $^{1} \cdot$ Bin Zhang ${ }^{1}$
}

Received: 30 August 2020 / Accepted: 8 November 2020 / Published online: 3 January 2021

(c) The Author(s), under exclusive licence to Springer-Verlag GmbH, AT part of Springer Nature 2021

\begin{abstract}
To investigate the viral communities in diarrhoeal faeces of Tibetan pigs, 146 diarrhoeic samples were collected from 16 pigs farms on the Tibetan plateau. Nineteen viruses belonging to eleven viral taxonomic families were identified in a pooled library. Metagenomics analysis revealed that the viruses were mainly small linear and circular DNA viruses. Furthermore, sequences of 10 NS1 genes and two complete genomes of PBuVs were obtained by PCR amplification. Sequence comparisons and phylogenetic analysis showed that the PBuVs from Tibetan pigs displayed more abundant genetic diversity than those from domestic pigs. This is the first description of the faecal viral community in Tibetan pigs associated with diarrhoea.
\end{abstract}

The Tibetan pig is a unique Chinese indigenous pig breed that is found on the Tibetan plateau of China [1]. Due to the natural and harsh environment with an average altitude of over 3,000 $\mathrm{m}$ above sea level, few viral infections have been reported in animals in this region until recently. Tibetan pigs graze in the pastures in a free-range system and are usually kept together with other livestock such as yaks (Bos grunniens), sheep, and goats, providing opportunities for viruses to circulate among the livestock. Animals on the Tibetan plateau are also kept in close contact with local humans, which increases the risk of zoonotic transmission. Viral diarrhoea is a common disease in Tibetan pigs that substantially increases the mortality and morbidity of piglets. Currently, only two viruses that can cause diarrhoeal disease in Tibetan pigs have been identified: porcine epidemic diarrhea virus

Handling Editor: Sheela Ramamoorthy.

Long Zhou and Chengzhe Hu contributed equally to this work.

Supplementary Information The online version contains supplementary material available at https://doi.org/10.1007/s0070 5-020-04932-9.

Bin Zhang

binovy@sina.com

1 College of Animal and Veterinary Sciences, Southwest Minzu University, Chengdu 610041, P.R. China

2 Institute of Animal Science of Ganzi Tibetan Autonomous Prefecture of Sichuan Province, Kangding 626000, P.R. China
(PEDV) [2, 3] and porcine deltacoronavirus (PDCoV) [4]. The identities of other viruses associated with diarrhoea in Tibetan pigs remain unclear.

Since the advent of high-throughput sequencing and new metagenomic analytical methods, viral metagenomics has shown a great potential for investigating unknown aetiologies and identifying novel viruses [5]. Since 2007, numerous studies on the discovery of new viral communities in humans and animals by virome sequencing have been reported [6]. In recent years, the approach has been successful for analysing the viromes and identifying viruses in serum, plasma, faeces, respiratory secretions, and tissue samples. The faecal virome has been characterized for various animals, and some new viruses associated with diarrhoea have been identified in domestic pigs, including PDCoV [7], swine enteric alphacoronavirus ( $\mathrm{SeACoV}$ ) [8], swine acute diarrhea syndrome coronavirus (SADS-CoV) [9], calicivirus [10], sapelovirus [11], and astrovirus (AstV) [12]. The existence of new viruses associated with diarrhoea in pigs is worthy of continuous investigation. Whether the above viruses are prevalent in Tibetan pigs at high altitude on the Tibetan plateau of China also deserves special attention.

From 2018 to 2019, a total of 146 faecal samples were collected from 10- to 40-day-old Tibetan piglets that had been associated with clinical diarrhoeal disease. These samples were obtained from 16 pig farms in Ganzi Tibetan autonomous prefecture of Sichuan province, which is located in the southeastern margin of the Tibetan plateau. Tibetan pigs are usually fed in a free-ranging system on the plateau. These animals are not vaccinated with any commercial 
vaccines, including live attenuated vaccines against porcine epidemic diarrhea virus or transmissible gastroenteritis virus. Soon after sampling, $10 \%$ (wt/vol) faecal suspensions were prepared using sterile phosphate-buffered saline (PBS). Before nucleic acids were extracted, these samples were subjected to a series of pretreatments as described previously [13]. Total viral RNA was prepared from the samples using a QIAamp Viral RNA Mini Kit (QIAGEN, Germany) according to the manufacturer's instructions. Then cDNA was generated using SuperScript III reverse transcriptase (RT) (Invitrogen, USA) and random hexamers (Invitrogen, USA) following the supplier's guidelines. Finally, one mixed pooled sample was sent to BGI Co. LTD (Shenzhen, China) for the library construction and high-throughput TruSeq Illumina sequencing.

Using high-throughput sequencing, a total of 884,471 reads were obtained from one pooled faecal sample of Tibetan pigs (GenBank accession number: SRR9276460). Approximately $7.2 \%$ (12,291 reads) of the sequence reads mapping to mammalian-associated viral sequences were recovered from the faecal samples. Using BLASTx software, members of eleven virus families (Fig. 1a) were identified, which, in order of sequence read abundance, were as follows: Smacoviridae (31.09\% of all reads), Parvoviridae (21.50\%), Genomoviridae (11.77\%), Hepeviridae (10.58\%), Adenoviridae (10.20\%), Picornaviridae (8.30\%), Circoviridae (2.82\%), Astroviridae (2.33\%), Coronaviridae (0.81\%), Flaviviridae $(0.33 \%)$, and Caliciviridae $(0.27 \%)$. Nineteen distinct viruses in these eleven families were identified (Fig. 1b). In order of sequence read abundance, the viruses were as follows: porcine stool-associated circular virus type
7 (PsaCV-7, 31.09\% of all reads), porcine bocavirus (PBoV, $16.80 \%$ ), gemycircularvirus (11.77\%), hepatitis E virus (HEV, 10.58\%), porcine adenovirus (10.20\%), enterovirus (4.46\%), sapelovirus (3.04\%), porcine circovirus 2 (PCV-2, $2.82 \%$ ), porcine parvovirus type 6 (PPV-6, $2.51 \%$ ), AstV (2.33\%), PPV-2 (1.33\%), porcine bufavirus $(0.86 \%)$, PEDV $(0.81 \%)$, posavirus $(0.41 \%)$, bovine viral diarrhea virus type 1 (BVDV-1, 0.33\%), porcine kobuvirus $(0.29 \%)$, sapovirus $(0.27 \%)$, rabovirus $(0.08 \%)$, and pasivirus $(0.02 \%)$. Three viruses in the study were demonstrated to be associated with animal diarrhoeal disease, including PEDV [14], porcine circovirus 2 (PCV-2) [15], and bovine viral diarrhea virus type 1 (BVDV-1) [16]. Interestingly, BVDV is frequently detected in cows, pigs, sheep, goats, and other ruminants [17], suggesting potential interspecies transmission of BVDV-1 between cattle and swine. It is noteworthy that HEV is considered to be a zoonotic pathogen, and cross-species transmission from animal reservoirs to humans is believed to be the major route of HEV transmission. In this study, the high sequence read abundance of HEV (10.58\%) indicates a high risk of zoonotic transmission between humans and Tibetan pigs. This is in agreement with previous reports $[18,19]$. In addition, three novel viruses, including porcine bufavirus, rabovirus, and pasivirus, may also be associated with diarrhoeal disease, which requires further investigation.

A total of 10 out of the 146 faecal samples tested positive for the NS1 gene by PCR amplification (6.85\% positive) (Table S1). All of the NS1 genes obtained in this study were 1738-1741 nt in length. Sequence alignment using the MegAlign program of DNAstar package version 7.0 (https:// www.dnastar.com/) indicated that they shared 95.6\%-96.3\% (a)

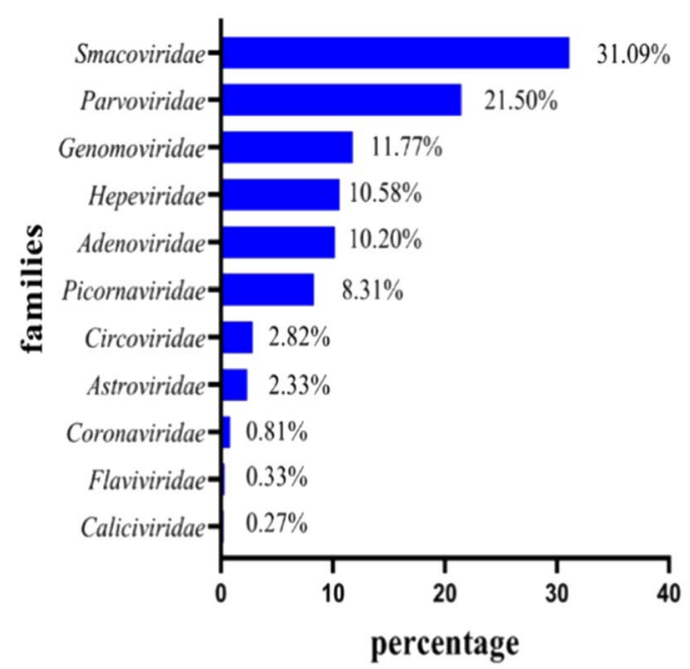

(b)

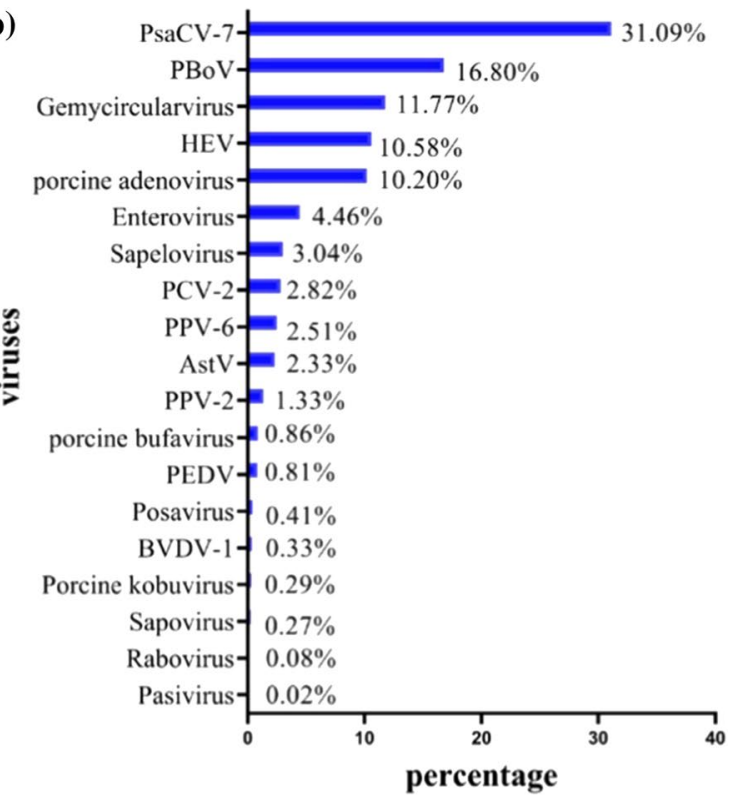

Fig. 1 Family (a) and species (b) classification and percentage of virus sequences detected from diarrhoeic fecal samples from Tibetan pigs 
nucleotide sequence identity with each other and were $90.7 \%-99.7 \%$ identical to the NS1 sequences of reference strains from pigs; however, they were only $43.9 \%-60.1 \%$ identical to those of reference strains originating from other species, including bats, canines, rats, and humans. An amino acid sequence alignment showed that the amino acid sequence identity among the ten NS1 genes ranged from $94.8 \%$ to $100 \%$ and that they shared $92.3 \%-99.3 \%$ amino acid sequence identity with other reference strains originating from domestic pigs. Interestingly, the 19GZ211 strain had a deletion of one amino acid at position 522 (Fig. S1), the same aa deletion that was found in the recently identified Henan019 strain in Henan province [20], more than a thousand kilometres away from the location of the Tibetan pig farms sampled in this study.

Phylogenetic analysis indicated that all of the NS1s in this study clustered in the swine-origin group, together with other bufaviruses that were identified in pigs. Notably, except for 18GZ201, which was found to be closely related to a PBuV (61/Austria/2014) identified in Austria in 2014 [21], nine of the ten strains clustered together with viruses from mainland China that are widely distributed in Guangxi, Guangdong, Fujian, Anhui, and Henan provinces [20] (Fig. 2a). The data in the present study show that the $\mathrm{PBuVs}$ from Tibetan pigs display abundant genetic diversity.

The full-length genomic sequences of two strains (designated SMU-TP-YN18 and SMU-TP-GZ18) were successfully amplified from samples that were positive for bufavirus. These sequences have been submitted to the GenBank database (accession no. MN868670 and MN868671, respectively), and both genome sequences were 4,189 nucleotides in length. Analysis using the MegAlign program of the DNAstar package version 7.0 (https ://www.dnastar.com/) revealed $96.0 \%$ nucleotide sequence identity between SMU-TP-YN18 and SMU-TP-GZ18 and $94.9 \%$ to $98.3 \%$ identity to reference genome sequences of bufaviruses of domestic pigs, but only $52.2 \%$ to $63.6 \%$ identity to reference strains from other species (Table 1).

To examine the genetic variation between the two $\mathrm{PBuVs}$, the nucleotide and deduced amino acid (aa) sequences of the NS1, VP1, SHP, and VP2 genes of these two isolates as well as three strains (FJNP2018, Anhui021, and 61/Austria/2014) from domestic pigs and four strains (BJ181, CBuV-88, MAG12-57, SY-2015) from humans, canines, bats, and rats, respectively, were compared. The results showed that NS1, VP1, SHP, and VP2 of SMU-TPYN18 shared 95.6\%-98.9\% nucleotide (91.4\%-97.4 aa) sequence identity with FJNP2018, a higher percentage than with the other reference strains. Meanwhile, NS1, VP1, SHP, and VP2 of the SMU-TP-GZ18 strain shared 98.6\%-99.2\% nucleotide (96.0\%-99.5 aa) sequence identity with 61/Austria/2014, which was higher than that shared with other six PBuVs (Table 1). These data indicate

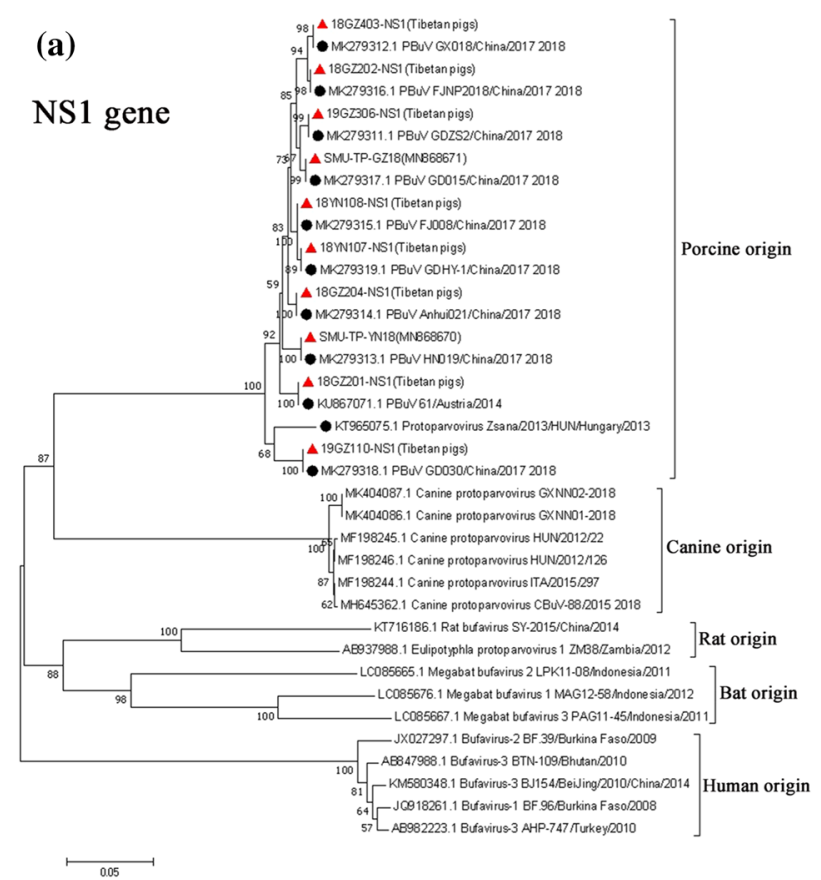

Fig. 2 Phylogenetic tree based on NS1 gene (a) and full-length genomic sequences (b) of the bufavirus strains identified in Tibetan pigs and other reference strains. The strains identified in this study are indicated by red triangles. The reference bufaviruses originating from

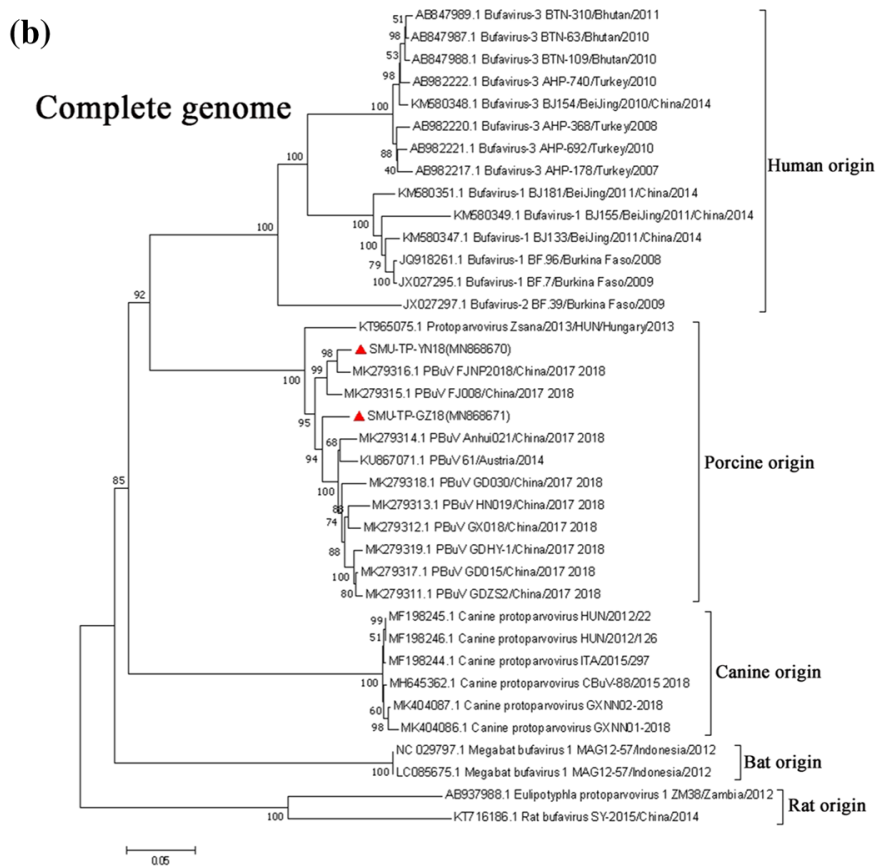

domestic pigs are indicated by black circles. The phylogenetic trees were constructed by the neighbor-joining method with 1,000 replicates using the MEGA7 program (http://www.megasoftware.net). The scale bar indicates the number of nucleotide substitutions per site. 
Table 1 Genome positions, protein sizes, and nucleotide and amino acid sequence identity values for different regions of the SMU-TP-YN18 genome and SMU-TP-GZ18 compared with bufavirus reference strains

\begin{tabular}{|c|c|c|c|c|c|c|c|c|c|}
\hline \multirow[t]{3}{*}{ Region } & \multirow{2}{*}{\multicolumn{2}{|c|}{$\frac{\text { Length (nt/aa) }}{\text { Tibetan pig origin }}$}} & \multicolumn{7}{|c|}{ Pairwise $\%$ identity (nt/aa) } \\
\hline & & & \multicolumn{3}{|c|}{ Domestic pig origin $^{1}$} & \multirow{2}{*}{$\begin{array}{l}\text { Human } \\
\text { origin }^{1} \\
\text { BJ181 }\end{array}$} & \multirow{2}{*}{$\begin{array}{l}\text { Canine } \\
\text { origin }^{1} \\
\text { CBuV-88 }\end{array}$} & \multirow{2}{*}{$\begin{array}{l}\text { Bat origin }^{1} \\
\text { MAG12-57 }\end{array}$} & \multirow{2}{*}{$\begin{array}{l}\text { Rat origin }{ }^{1} \\
\text { SY-2015 }\end{array}$} \\
\hline & $\begin{array}{l}\text { SMU-TP- } \\
\text { YN18 }\end{array}$ & $\begin{array}{l}\text { SMU-TP- } \\
\text { GZ18 }\end{array}$ & FJNP2018 & Anhui021 & $\begin{array}{l}\text { 61/Aus- } \\
\text { tria/2014 }\end{array}$ & & & & \\
\hline \multirow{2}{*}{$\begin{array}{r}\text { Complete } \\
\text { genome }\end{array}$} & \multirow[t]{2}{*}{4189} & \multirow[t]{2}{*}{4189} & $97.4 *$ & $95.4^{*}$ & $94.9 *$ & $62.9 *$ & $63.5^{*}$ & $58.7^{*}$ & $52.2 *$ \\
\hline & & & $94.9 * *$ & $98.0 * *$ & $98.3 * *$ & $63.2 * *$ & $63.6 * *$ & $58.2^{* *}$ & $52.2 * *$ \\
\hline \multirow[t]{2}{*}{ NS1 } & \multirow[t]{2}{*}{$1741 / 579$} & \multirow[t]{2}{*}{$1741 / 579$} & $98.7 / 97.4$ & $98.4 / 96.0$ & $97.3 / 92.8$ & $44.4 / 36.3$ & $59.4 / 37.8$ & $57.1 / 37.6$ & $57.3 / 36.3$ \\
\hline & & & $97.3 / 93.8$ & $98.2 / 95.6$ & $99.2 / 96.0$ & $44.8 / 34.2$ & $60.2 / 38.4$ & $57.4 / 37.5$ & $57.4 / 36.3$ \\
\hline \multirow[t]{2}{*}{ VP1 } & \multirow[t]{2}{*}{$2035 / 679$} & \multirow[t]{2}{*}{$2035 / 679$} & 97.2/93.4 & $93.3 / 91.4$ & 93.2/91.4 & $73.0 / 67.7$ & $70.4 / 67.5$ & $64.3 / 56.6$ & $57.3 / 50.3$ \\
\hline & & & 93.2/91.4 & $97.8 / 97.6$ & 98.6/98.4 & $72.6 / 68.6$ & $69.7 / 67.8$ & $64.3 / 57.9$ & $56.7 / 49.9$ \\
\hline \multirow[t]{2}{*}{ SHP } & \multirow[t]{2}{*}{$389 / 129$} & \multirow[t]{2}{*}{$389 / 129$} & 98.9/91.4 & $86.7 / 81.5$ & $86.2 / 82.3$ & - & - & - & - \\
\hline & & & $86.2 / 82.3$ & $96.7 / 98.5$ & 98.8/99.5 & - & - & - & - \\
\hline \multirow[t]{2}{*}{ VP2 } & \multirow[t]{2}{*}{$1613 / 538$} & \multirow[t]{2}{*}{$1613 / 538$} & 95.6/93.4 & $92.6 / 83.9$ & $92.3 / 84.2$ & $72.6 / 53.5$ & $69.0 / 46.6$ & $63.6 / 43.5$ & $56.0 / 34.3$ \\
\hline & & & $92.6 / 84.2$ & $97.7 / 94.3$ & 99.2/97.6 & $72.0 / 51.8$ & $68.0 / 44.7$ & $63.0 / 42.3$ & $55.4 / 34.3$ \\
\hline
\end{tabular}

${ }^{1}$ In each box, the upper figure is the percentage identity to SMU-TP-YN18 $(*)$ and the lower figure is the percentage identity to SMU-TP-GZ18 (**). The highest nucleotide and amino acid sequence identities of different regions are indicated in bold typeface. "-" indicates the lack of an SHP gene in bufavirus originating from humans, canines, bats, and rats.

that the two PBuVs in this study are closely related to $\mathrm{PBuVs}$ that originated from domestic pigs.

Further examination of the phylogenetic tree based on whole genome sequences revealed that the SMU-TP-YN18 strain from this study showed a close relationship to the FJNP2018 strain, which was recently identified in Fujian province of China [20]. The SMU-TP-GZ18 strain occupied a separate branch with $94 \%$ bootstrap support (Fig. 2b), suggesting a new evolution direction for this strain.

Bufaviruses are newly discovered zoonotic viruses that have been reported in numerous mammals and humans [22]. They are single-stranded non-segmented DNA viruses belonging to the genus Protoparvovirus, subfamily Parvoviridae (http://ictvonline.org). The bufavirus genome is about $4-6 \mathrm{~kb}$ in length and encodes a non-structural protein 1 (NS1), a putative structural protein 1 (VP1), a small hypothetical protein (SHP), and structural protein 2 (VP2) [22-24]. The virus was first detected in faecal samples from children with acute diarrhoea in Burkina Faso in 2012 [25]. Sporadic cases of diarrhoea with shedding of bufavirus have been reported in several countries, suggesting that the virus might be a possible occasional cause of diarrhoea in humans. To date, bufaviruses have been detected in humans and mammals such as rats, shrews, pigs, dogs, bats, and primates, implying a broad species host tropism with a high risk of interspecies transmission. Therefore, these viruses deserve continuous monitoring and special attention for control.

In conclusion, the results of the present study revealed 19 distinct viruses belonging to 11 viral taxonomic families, extending our knowledge of viral communities associated with porcine diarrhoeal disease for the first time to Tibetan pigs. Sequence comparisons and phylogenetic analysis based on the NS1 gene and whole-genome sequences of PBuVs indicated that the viruses in Tibetan pigs were more genetically diverse than those originating in Chinese domestic pigs. Because bufaviruses are newly discovered zoonotic viruses that can infect various mammals and humans, surveillance of $\mathrm{PBuVs}$ is of great significance for veterinary and public health.

Acknowledgements This work was supported financially by the National Natural Science Foundation of China (grant no. 31772766), the Sichuan Science and Technology Program (grant no. 2020YJ0247), the Application Fundamental Research Program of Ganzi Tibetan Autonomous Prefecture (grant no. 20yyjs0030), and the Fundamental Research Funds for the Central Universities of Southwest Minzu University (grant no. 2020NQN30).

\section{Compliance with ethical standards}

Conflict of interest The authors declare that they have no conflict of interest.

\section{References}

1. Li M, Tian S, Jin L, Zhou G, Li Y, Zhang Y, Wang T, Yeung CK, Chen L, Ma J, Zhang J, Jiang A, Li J, Zhou C, Zhang J, Liu Y, Sun X, Zhao H, Niu Z, Lou P, Xian L, Shen X, Liu S, Zhang S, Zhang M, Zhu L, Shuai S, Bai L, Tang G, Liu H, Jiang Y, Mai M, Xiao J, Wang X, Zhou Q, Wang Z, Stothard P, Xue M, Gao X, Luo Z, Gu Y, Zhu H, Hu X, Zhao Y, Plastow GS, Wang J, Jiang Z, 
Li K, Li N, Li X, Li R (2013) Genomic analyses identify distinct patterns of selection in domesticated pigs and Tibetan wild boars. Nat Genet 45:1431-1438

2. Gao X, Zhang L, Jiang X, Mehmood K, Wang L, Tong X, Wang M, Zhang H, Li J (2019) Porcine epidemic diarrhea: an emerging disease in Tibetan pigs in Tibet, China. Trop Anim Health Prod 51:491-494

3. Qin S, Hu C, Yang D, Wu J, Yue H, Tang C, Zhang B (2019) Emergence of porcine epidemic diarrhea viruses with the novel $\mathrm{S}$ genes in Tibetan pigs in the Qinghai-Tibetan plateau in China. Virus Res 270:197652

4. Wang M, Wang Y, Baloch AR, Pan Y, Tian L, Xu F, Shivaramu S, Chen S, Zeng Q (2018) Detection and genetic characterization of porcine deltacoronavirus in Tibetan pigs surrounding the QinghaiTibet Plateau of China. Transbound Emerg Dis 65:363-369

5. Edwards RA, Rohwer F (2005) Viral metagenomics. Nat Rev Microbiol 3:504-510

6. Delwart EL (2007) Viral metagenomics. Rev Med Virol 17:115-131

7. Hu H, Jung K, Vlasova AN, Chepngeno J, Lu Z, Wang Q, Saif LJ (2015) Isolation and characterization of porcine deltacoronavirus from pigs with diarrhea in the United States. J Clin Microbiol 53:1537-1548

8. Pan Y, Tian X, Qin P, Wang B, Zhao P, Yang YL, Wang L, Wang D, Song Y, Zhang X, Huang YW (2017) Discovery of a novel swine enteric alphacoronavirus $(\mathrm{SeACoV})$ in southern China. Vet Microbiol 211:15-21

9. Zhang F, Luo S, Gu J, Li Z, Li K, Yuan W, Ye Y, Li H, Ding Z, Song D, Tang Y (2019) Prevalence and phylogenetic analysis of porcine diarrhea associated viruses in southern China from 2012 to 2018. BMC Vet Res 15:470

10. Fan K, Wang R (2012) Complete genome of a porcine calicivirus strain in Anhui province, China, is significantly shorter than that of the other Chinese strain. J Virol 86:13823

11. Chen J, Chen F, Zhou Q, Li W, Song Y, Pan Y, Zhang X, Xue C, Bi Y, Cao Y (2012) Complete genome sequence of a novel porcine Sapelovirus strain YC2011 isolated from piglets with diarrhea. J Virol 86:10898

12. Reuter G, Pankovics P, Boros A (2011) Identification of a novel astrovirus in a domestic pig in Hungary. Arch Virol 156:125-128

13. Zhang B, Tang C, Yue H, Ren Y, Song Z (2014) Viral metagenomics analysis demonstrates the diversity of viral flora in piglet diarrhoeic faeces in China. J Gen Virol 95:1603-1611

14. Wang D, Fang L, Xiao S (2016) Porcine epidemic diarrhea in China. Virus Res 226:7-13
15. Wang H, Gu J, Xing G, Qiu X, An S, Wang Y, Zhang C, Liu C, Gong W, Tu C, Su S, Zhou J (2019) Genetic diversity of porcine circovirus type 2 in China between 1999-2017. Transbound Emerg Dis 66:599-605

16. Hou P, Zhao G, Wang H, He H (2019) Prevalence of bovine viral diarrhea virus in dairy cattle herds in eastern China. Trop Anim Health Prod 51:791-798

17. Deng Y, Wang S, Liu R, Hao G (2018) Genetic diversity of bovine viral diarrhea virus infection in goats in Southwestern China. J Vet Med 2018:8274397

18. Zhang L, Huang S, Li K, Rehman MU, Jiang X, Tong X, Zhang H, Iqbal MK, Mehmood K, Liu S, Shen Y, Li J (2018) Molecular detection of indigenous Hepatitis E virus (HEV) from Tibetan Pigs in Tibet, China. Food Environ Virol 10:373-377

19. Zhou K, Zhou Q, Yang D, Yue H, Tang C, Zhang B (2019) Hepatitis $\mathrm{E}$ virus in Tibetan pigs on the Qinghai-Tibetan Plateau: detection, molecular characterization and infection of BALB/c mice. Arch Virol 164:2943-2951

20. Sun YK, Chen YJ, Cai Y, Zhu DH, Pan HM, Wei YF, Han XL, Ji CH, Lu G, Wang H, Ma CQ, Zhang GH (2020) First report and genetic diversity of porcine bufavirus in China. Virol J 17:2

21. Liu L, Schwarz L, Ullman K, Ahola H, Qiu Y, Ma Z, HennigPauka I (2016) Identification of a novel bufavirus in domestic pigs by a viral metagenomic approach. J Gen Virol 97:1592-1596

22. Chieochansin T, Vutithanachot V, Theamboonlers A, Poovorawan Y (2015) Bufavirus in fecal specimens of patients with and without diarrhea in Thailand. Arch Virol 160:1781-1784

23. Kemenesi G, Dallos B, Gorfol T, Estok P, Boldogh S, Kurucz K, Oldal M, Marton S, Banyai K, Jakab F (2015) Genetic diversity and recombination within bufaviruses: detection of a novel strain in Hungarian bats. Infect Genet Evol 33:288-292

24. Li J, Cui L, Deng X, Yu X, Zhang Z, Yang Z, Delwart E, Zhang W, Hua X (2019) Canine bufavirus in faeces and plasma of dogs with diarrhoea, China. Emerg Microbes Infect 8:245-247

25. Altay Kocak A, Ocal M, Polat M, Kanik Yuksek S, Aktas Tapisiz A, Tezer H, Ozkul A, Ergunay K, Bozdayi G, Ahmed K (2017) Multicenter investigation of bufavirus in the etiology of viral central nervous system infections of adults and children. Mikrobiyol Bul 51:191-194

Publisher's Note Springer Nature remains neutral with regard to jurisdictional claims in published maps and institutional affiliations. 